\section{Effect of Thiobacillus and superabsorbent on essential oil components in Thyme species}

1

2

3

Pouneh Pouramini ${ }^{1,4}$, Mohammad Hossein Fotokian ${ }^{2}$, Hossein Dehghan $^{3}$, Goetz Hensel ${ }^{4 *}$ 4

${ }^{1}$ Horticulture Department, Faculty of Agriculture Sciences, Karaj University, Karaj, Iran 5

${ }^{2}$ Agricultural College, Shahed University, Tehran, Iran.

6

7

${ }^{3}$ Medicinal Plants Research Center, Shahed University, Tehran, Iran.

8

${ }^{4}$ Leibniz Institute of Plant Genetics and Crop Plant Research (IPK), Gatersleben, Germany. $\quad 9$

*Corresponding author:

Dr. Goetz Hensel

E-mail: hensel@ipk-gatersleben.de 15

Tel: +49 39482-5543 16

Fax: +49 39482-5515 
$\begin{array}{ll}\text { Abstract } & 20\end{array}$

Optimal nutrition along with non-stress conditions has a significant impact on the quantity

and quality of essential oil in medicinal plants. The objective of this research was to examine

the possibility of improving the quantity and quality of essential oil in thyme through nutrition of the seedlings using Thiobacillus bio-fertilizer and treatment by Superabsorbent.

were sown in pots and exposed to different levels of Thiobacillus and superabsorbent. Results

of Gas Chromatography Mass Spectrometry (GC/MS) revealed that the main compounds

detected for T. vulgaris were thymol (31.5\%), p-cymene (23.4\%), $\gamma$-terpinene (13.9\%),

linalool $(38.3 \%)$ and carvacrol $(2.7 \%)$ while the main compounds of $T$. daenensis were

thymol $(51.2 \%)$, o-cymene (12.9\%), $\gamma$-terpinene (4.5\%), linalool (1.7\%) and borneol $(3.1 \%)$.

Furthermore, the application of Thiobacillus had a significant effect on $\alpha$-pinene content and caryophyllene.

Keywords: borneol, carvacrol, caryophyllene, thymol, thyme 


\section{Introduction}

The genus Thymus, with about 215 species, is one of the eight most important genera

of the Lamiacea family. Thyme, a plant native to the Mediterranean region (Spain, Italy,

France, Greece, etc.), has long been used as a source of essential oil and other constituents

(e.g. thymol, flavanoid, caffeic acid and labiatic acid) derived from the different parts of the

plant. In addition to their numerous traditional uses, the plant (herb) and its essential oil have

found diverse applications in pharmacy and medicine [1]. Thymus vulgaris is a perennial

medicinal plant, cultivated worldwide for a lot of uses like culinary, cosmetic and medical

purposes. This species has special activities such as antispasmodic, expectorant, antiseptic,

Depending on the growing conditions (different environments, climates, soil,

Thyme contains 0.8 to $2.6 \%$ (generally $1 \%$ ) of essential oils which is primarily 
microbial inoculants consisting of living cells of microorganism like bacteria, algae and fungi

alone or combination which can help in increasing crop productivity. Organic manures can

serve as alternative to mineral fertilizers for improving soil structure, soil nutrients, soil

physical and chemical characteristics, increase soil fertility and plant plants resistance to

diseases and salt stress, decrease plant diseases, improving crop growth, development, yield

$[10,11]$. through organic and biological methods plays a key role in increasing qualitative and

In many regions of Iran, soil type is calcareous with high $\mathrm{pH}$. Therefore, some nutrients such as phosphorus, iron and zinc are stabilized and non-absorbable for plants. In such situations, Thiobacillus bacteria can reduce the $\mathrm{pH}$ of the soil in the presence of sulfur resulting in the solubility of nutrients around the root area [13].

These previously mentioned issues have increased along with limited water resources in the arid and semi-arid ecosystems of Iran [14]. Water stress has many adverse effects on and quality of active ingredients while these herbs need to perfect vegetative and have been defined as polymeric materials which exhibit the ability of swelling in water and retaining a significant fraction (>20\%) of water within their structure, without dissolving in 
yet been examined on medicinal plants. Therefore, this research was conducted to investigate

the possibility of improving the quantity and quality of essential oil in Thyme through

nutrition of the seedlings by Thiobacillus bio-fertilizer and increase of available water

The experiment was conducted in a greenhouse on a research farm of an Agricultural

A factorial experiment using a completely randomized design with two replications

The seeds were planted on 5 April 2011 and germinated after 17 days. The first flower 
Statistical analyses were conducted using SPSS version 16.0. A normality test of

\section{Results and discussion}

The Thymus vulgaris essential oil included 31 constituents which represented $96.7 \%$

of the total analyzed oil. The main compounds included thymol (31.5\%), p-cymene $(23.4 \%)$,

$\gamma$-terpinene (13.9\%), linalool (3.4\%), thymol methyl ether $(3.6 \%)$ and carvacrol $(2.7 \%)$.

Further compounds and their constituents are presented in Table 2. In contrast, the Thymus

daenensis species contained 33 compounds in the essential oil which represented $96.19 \%$ of

the total analyzed oil. The main compounds included thymol (51.18\%), O-cymene $(12.87 \%)$,

The components identified in Thymus vulgaris are in accordance with a study 
previous published data. The reason for that might be the different soil characteristics because

Extractable oil composition strongly depends on the method used. In a study by

Charles and Simon [21] comparison of different extraction methods showed that hydro

Analysis of variance of some components of thyme essential oil is presented in Table

3. The difference between two thyme species was significant $(P \leq 0.01)$ for all of the

A review of the literature regarding the application of Thiobacillus showed that there was little information about the use on medicinal plants. In a recent study, the effect of Bio- 
microbial inoculum application on the yield and nutrients content of Dracocephalum

moldavica L. seeds was evaluated. In a field experiment, the highest yield was obtained by

strains) improves the iron nutrient content of the seeds [21]. Also, in the presence of sulfur,

Thiobacillus increased nutrient uptake, growth and production of Melissa officinalis oil compared to the control [12].

Razban and Pirzad [16] investigated the effect of superabsorbent on Matricaria

\subsection{Relations between the main compounds of essential oil}

Pearson's correlation coefficients between traits showed a significant positive effects.

\subsubsection{Superabsorbent $\times$ Thiobacillus interaction}

Combined levels of the superabsorbent $\times$ Thiobacillus interaction were significant for borneol

and caryophyllene (Table 4 and Figure $1 ; P \leq 0.05$ ). The maximum amount of borneol was

observed in $\mathrm{S}_{0} \mathrm{~T}_{0}$ treatment, although there was no significant difference in the amount of

borneol in the treatment $S_{1} T_{0.5}$. Although the maximum amount of caryophyllene was

statistically significant (Figure 1). The differences among superabsorbent levels were not 
difference among levels of superabsorbent. With the increase of the content in superabsorbent treatment levels, the differences among these levels may be significant for essence components.

\subsubsection{Species $\times$ Thiobacillus interaction}

The interaction between species $\times$ Thiobacillus was significant for thymol $(P \leq 0.05$; Table

4). Using Thiobacillus in Thymus daenensis led to a significant reduction in the amount of

\subsubsection{The triple interaction among species $\times$ superabsorbent $\times$ Thiobacillus}

Significant differences among combinations of this interaction were observed only for

Borneol and caryophyllene $(P \leq 0.05)$ (Table 6$)$. The maximum amount of caryophyllene was

obtained with Thymus daenensis in the $\mathrm{S}_{0.5} \mathrm{~T}_{0.5}$ treatment, with no statistically significant

not improve the amount of borneol. The amount of borneol in Thymus vulgaris was

The maximum amount of borneol was observed in the $\mathrm{S}_{0} \mathrm{~T}_{0}$ treatment, although there was no

The interaction between species $\times$ superabsorbent was not significant for all traits, while for species main factor it was significant at $P \leq 0.01$. Referring to an acceptable amount of 
The correlation between thymol with others was negatively significant, with the exception of

a positive correlation with caryophylene. In conclusion, thymol is the most important part of

essence component in Thymus; any trying to increase thymol content will decrease other

\section{Conclusion}

The interaction between Thiobacillus and superabsorbent on oil and essential oil yield was

was higher than Thymus daenensis. Despite the fact that Thymus vulgaris is not native to Iran,

its high value in the pharmaceutical, cosmetic, health and culture, food and export will

encourage its cultivation. In addition, the variance analysis of our data showed the amount of

all components in $T$. vulgaris are much higher than $T$. daenensis, with the exception of 
Tables

Table 1. Selected physical and chemical properties of soil used in this study.

\begin{tabular}{lcccccccc}
\hline Tissue & $\mathbf{P}$ & $\mathbf{K}$ & $\begin{array}{c}\text { Total } \\
\text { Nppm] }\end{array}$ & $\begin{array}{c}\text { Organic } \\
\text { [ppm] } \\
{[\%]}\end{array}$ & $\begin{array}{c}\text { Calcium } \\
\text { matter } \\
\text { carbonate } \\
\text { equivalent } \\
{[\%]}\end{array}$ & $\mathbf{p H}$ & $\begin{array}{c}\mathbf{E C} \\
{[\mathbf{d s} / \mathbf{m}]}\end{array}$ & $\begin{array}{c}\text { Percent } \\
\text { saturation } \\
{[\%]}\end{array}$ \\
\hline Loamy & 38 & 344.7 & 0.22 & 2.19 & 7.5 & 8.26 & 1.32 & 45 \\
\hline
\end{tabular}

Table 2. Compounds identified in Thymus vulgaris and Thymus daenensis

\begin{tabular}{|c|c|c|c|c|c|c|}
\hline & & Thymus $v$ & lgaris & Thymus & enensis & \\
\hline No. & Compound & RT [min] & {$[\%]$} & RT [min] & {$[\%]$} & Class \\
\hline 1 & $\alpha$-Thujene & $11: 12$ & 1.51 & $11: 05$ & 0.90 & $\mathrm{MH}$ \\
\hline 2 & $\alpha$-Pinene & $11: 44$ & 1.17 & $11: 35$ & 0.68 & $\mathrm{MH}$ \\
\hline 3 & Camphene & $12: 10$ & 0.78 & $12: 05$ & 0.52 & $\mathrm{MH}$ \\
\hline 4 & $\beta$-Pinene & $13: 49$ & 0.36 & $13: 44$ & 0.30 & $\mathrm{MH}$ \\
\hline 5 & Octen-3-ol & - & - & $13: 86$ & 0.23 & Others \\
\hline 6 & $\beta$-Myrcene & $14: 39$ & 3.32 & $14: 32$ & 0.88 & $\mathrm{MH}$ \\
\hline 7 & Phellandrene & & & $14: 91$ & 0.18 & $\mathrm{MH}$ \\
\hline 8 & $\alpha$-Phellandrene & $15: 23$ & 0.44 & - & - & $\mathrm{MH}$ \\
\hline 9 & $p$-Cymene & $16: 90$ & 23.40 & - & - & $\mathrm{MH}$ \\
\hline 10 & $\alpha$-Terpinene & - & - & $15: 60$ & 1.14 & $\mathrm{MH}$ \\
\hline 11 & $o$-Cymene & - & - & $16: 31$ & 12.87 & $\mathrm{MH}$ \\
\hline 12 & 1,8-Cineole & - & - & $16: 52$ & 1.94 & MO \\
\hline 13 & $\gamma$-Terpinene & $18: 63$ & 13.94 & $17: 95$ & 4.48 & $\mathrm{MH}$ \\
\hline 14 & $\beta$-Terpineol & $18: 80$ & 0.87 & $18: 31$ & 0.84 & MO \\
\hline 15 & $\alpha$-Terpinolene & $19: 50$ & 0.25 & $19: 24$ & 0.15 & $\mathrm{MH}$ \\
\hline 16 & Linalool & $20: 47$ & 3.38 & $20: 06$ & 1.68 & MO \\
\hline 17 & Borneol & $23: 34$ & 1.02 & $23: 73$ & 3.09 & MO \\
\hline 18 & Terpinen-4-ol & $23: 85$ & 0.84 & - & - & MO \\
\hline 19 & Borneol & $24: 49$ & 1.61 & - & - & $\mathrm{MO}$ \\
\hline 20 & $\alpha$-Terpineol & - & - & $25: 76$ & 3.30 & MO \\
\hline 21 & Dihydro carvone & - & - & $26: 16$ & 0.13 & MO \\
\hline 22 & Thymol methyl ether & $26: 72$ & 3.58 & $26: 47$ & 1.19 & MO \\
\hline 23 & Carvacrol methyl ether & $27: 10$ & 1.65 & $26: 89$ & 0.58 & MO \\
\hline 24 & Thymoquinone & - & - & $28: 59$ & 3.02 & MO \\
\hline 25 & Thymol & $30: 62$ & 31.50 & $30: 64$ & 51.18 & MO \\
\hline 26 & Carvacrol & $31: 13$ & 2.66 & - & - & MO \\
\hline 27 & Caryophylene & $35: 06$ & 1.55 & $34: 96$ & 2.41 & $\mathrm{SH}$ \\
\hline 28 & Aromadenderene & - & - & $35: 67$ & 0.17 & $\mathrm{SH}$ \\
\hline 29 & $\alpha$-Humulene & - & - & $36: 28$ & 0.28 & $\mathrm{SH}$ \\
\hline 30 & Geranyl propionate & - & - & $37: 00$ & 0.12 & Others \\
\hline 31 & Geranyl propionate & $37: 13$ & 0.46 & - & - & Others \\
\hline 32 & $\alpha$-Amorphene & $37: 26$ & 0.12 & - & - & $\mathrm{SH}$ \\
\hline 33 & Germacrene D & $37: 46$ & 0.13 & - & - & $\mathrm{SH}$ \\
\hline 34 & Viridiflorene & - & - & $37: 95$ & 0.15 & $\mathrm{SH}$ \\
\hline 35 & Cubebol & $37: 99$ & 0.10 & - & - & $\mathrm{SH}$ \\
\hline
\end{tabular}




\begin{tabular}{lllllll}
\hline $\mathbf{3 6}$ & $\beta$-Bisabolene & - & - & $38: 47$ & $\mathbf{0 . 4 2}$ & SH \\
$\mathbf{3 7}$ & $\gamma$-Cadinene & $38: 77$ & $\mathbf{0 . 1 8}$ & $39: 05$ & $\mathbf{0 . 1 6}$ & SH \\
$\mathbf{3 8}$ & $\delta$-cadinene & $39: 13$ & $\mathbf{0 . 4 3}$ & - & - & SO \\
$\mathbf{3 9}$ & Thymohydro quinone & - & - & $40: 82$ & $\mathbf{1 . 1 8}$ & SO \\
$\mathbf{4 0}$ & Caryophelen oxide & $41: 54$ & $\mathbf{0 . 7 5}$ & $41: 52$ & $\mathbf{2 . 1 3}$ & SO \\
$\mathbf{4 1}$ & Eudesmol & $42: 87$ & $\mathbf{0 . 0 9}$ & - & - & SO \\
$\mathbf{4 2}$ & $\alpha$-epi-Cadinol & $43: 68$ & $\mathbf{0 . 2 1}$ & - & - & SO \\
$\mathbf{4 3}$ & $\alpha$-Cadinol & $44: 17$ & $\mathbf{0 . 1 0}$ & - & - & SO \\
$\mathbf{4 4}$ & (E)-14-Hydroxy-9-epi- & $44: 81$ & $\mathbf{0 . 1 3}$ & - & - & SO \\
& caryophyllene & - & - & $45: 20$ & $\mathbf{0 . 1 2}$ & Others \\
$\mathbf{4 5}$ & $\alpha$-epi-Bisabolol & $54: 87$ & $\mathbf{0 . 1 3}$ & $54: 84$ & $\mathbf{0 . 1 3}$ & Others \\
$\mathbf{4 6}$ & Hexadecanoic acid & - & - & $60: 20$ & $\mathbf{3 . 2 2}$ & MH \\
$\mathbf{4 7}$ & Abiatatriene & \multicolumn{5}{c}{$\mathbf{9 6 . 1 9}$} \\
\hline & Total Identified & $\mathbf{9 6 . 6 7}$ \\
\hline MH - monoterpene hydrocarbons; MO- oxygenated monoterpenes; SH-sesquiterpene hydrocarbons; SO- oxygenated sesquiterpenes
\end{tabular}


Table 3. Analysis of variance of the effects of superabsorbent and Thiobacillus on the essential oil of two species of thyme

\begin{tabular}{|c|c|c|c|c|c|c|c|c|c|c|c|}
\hline \multirow{2}{*}{ S.O.V } & \multirow{2}{*}{ df } & \multicolumn{10}{|c|}{ Mean squares } \\
\hline & & $\alpha$-Thujene & $\alpha$-Pinene & Camphene & $\beta$-Myrcene & $p$-Cymene & $\gamma$-Terpinene & Linalool & Borneol & Thymol & Caryophyllene \\
\hline $\mathrm{S}$ & 1 & $0.562^{* *}$ & $1.321^{* * 4}$ & $0.702^{*}$ & $11.323^{* * *}$ & $2110.034^{* * *}$ & $441.438^{* * 4}$ & $19.706^{* * * 4}$ & $3.683^{* *}$ & $2545.542^{* * *}$ & $28.423^{\text {*** }}$ \\
\hline SA & 2 & $0.024^{\mathrm{ns}}$ & $0.058^{\mathrm{ns}}$ & $0.038^{\mathrm{ns}}$ & $0.061^{\mathrm{ns}}$ & $7.561^{\mathrm{ns}}$ & $11.952^{\mathrm{ns}}$ & $0.005^{\mathrm{ns}}$ & $0.232^{\mathrm{ns}}$ & $74.919^{\mathrm{ns}}$ & $0.251^{\mathrm{ns}}$ \\
\hline $\mathrm{T}$ & 1 & $0.022^{\mathrm{ns}}$ & $0.003^{\mathrm{ns}}$ & $0.001^{\mathrm{ns}}$ & $0.018^{\mathrm{ns}}$ & $40.755^{*}$ & $0.832^{\mathrm{ns}}$ & $0.438^{\mathrm{ns}}$ & $0.061^{\mathrm{ns}}$ & $1.322^{\mathrm{ns}}$ & $0.295^{\mathrm{ns}}$ \\
\hline $\mathrm{T} \times \mathrm{SA}$ & 2 & $0.026^{\mathrm{ns}}$ & $0.054^{\mathrm{ns}}$ & $0.034^{\mathrm{ns}}$ & $0.001^{\mathrm{ns}}$ & $10.054^{\mathrm{ns}}$ & $4.751^{\mathrm{ns}}$ & $0.238^{\mathrm{ns}}$ & $0.481^{*}$ & $11.094^{\mathrm{ns}}$ & $0.559^{*}$ \\
\hline $\mathrm{S} \times \mathrm{SA}$ & 2 & $0.021^{\mathrm{ns}}$ & $0.030^{\mathrm{ns}}$ & $0.010^{\mathrm{ns}}$ & $0.075^{\text {ns }}$ & $0.319^{\mathrm{ns}}$ & $11.882^{\mathrm{ns}}$ & $0.338^{\mathrm{ns}}$ & $0.001^{\mathrm{ns}}$ & $41.655^{\mathrm{ns}}$ & $0.263^{\mathrm{ns}}$ \\
\hline $\mathrm{S} \times \mathrm{T}$ & 1 & $0.055^{\mathrm{ns}}$ & $0.107^{\mathrm{ns}}$ & $0.018^{\mathrm{ns}}$ & $0.002^{\mathrm{ns}}$ & $25.058^{\mathrm{ns}}$ & $0.695^{\mathrm{ns}}$ & $0.096^{\mathrm{ns}}$ & $0.034^{\mathrm{ns}}$ & $433.359^{*}$ & $0.112^{\mathrm{ns}}$ \\
\hline $\mathrm{S} \times \mathrm{T} \times \mathrm{SA}$ & 2 & $0.009^{\mathrm{ns}}$ & $0.026^{\mathrm{ns}}$ & $0.036^{\mathrm{ns}}$ & $0.008^{\mathrm{ns}}$ & $22.564^{\mathrm{ns}}$ & $5.611^{\mathrm{ns}}$ & $0.183^{\mathrm{ns}}$ & $0.384^{*}$ & $61.169^{\mathrm{ns}}$ & $0.469^{*}$ \\
\hline Error & 11 & 0.013 & 0.027 & 0.028 & 0.022 & 8.132 & 3.251 & 0.317 & 0.089 & 43.097 & 0.108 \\
\hline $\mathrm{CV} \%$ & - & 26.4 & 25.1 & 29.2 & 17.5 & 16.9 & 29.2 & 29.4 & 24.6 & 11.6 & 16 \\
\hline
\end{tabular}

Symbols: S, Species; SA, Superabsorbent; T, Thiobacillus.

$*$, ** significant differences at $\boldsymbol{P} \leq 0.05$ and $\boldsymbol{P} \leq 0.01$, respectively.

Table 4. Mean comparisons between two species for essence components by Duncan's multiple range test

\begin{tabular}{ccccccccccc}
\hline Species & $\alpha$-Thujene & $\alpha$-Pinene & Camphene & $\beta$-Myrcene & $p$-Cymene & $\gamma$-Terpinene & Linalool & Borneol & Thymol & Caryophyllene \\
\hline T. vulgaris & 0.583 & 0.887 & 0.743 & 1.529 & 26.181 & 9.192 & 2.823 & 1.603 & 46.297 & 0.964 \\
T. daenensis & 0.277 & 0.418 & 0.401 & 0.156 & 7.428 & 0.615 & 1.011 & 0.8197 & 66.895 & 3.141 \\
\hline
\end{tabular}

-Refer to variance analysis (Table 3), the differences between two Thymus species is statistically significant at $P \leq 0.01$

Table 5. Pearson's correlation coefficients between some essential components of two species of Thyme

\begin{tabular}{|c|c|c|c|c|c|c|c|c|c|}
\hline $\begin{array}{l}\text { Compound } \\
\alpha \text {-Pinene }\end{array}$ & $\begin{array}{l}\alpha \boldsymbol{\alpha} \text {-Thujene } \\
0.91^{* *}\end{array}$ & $\alpha$-Pinene & Camphene & $\beta$-Myrcene & p-Cymene & $\gamma$-Terpinene & Linalool & Borneol & Thymol \\
\hline Camphene & $0.782^{* *}$ & $0.848^{* *}$ & & & & & & & \\
\hline$\beta$-Myrcene & $0.748^{* *}$ & $0.781^{* *}$ & $0.701^{* *}$ & & & & & & \\
\hline$p$-Cymene & $0.789^{* *}$ & $0.798^{* * *}$ & $0.733^{* *}$ & $0.932^{* *}$ & & & & & \\
\hline$\gamma$-Terpinene & $0.76^{* * *}$ & $0.696^{* *}$ & $0.655^{* *}$ & $0.881^{* *}$ & $0.874^{* *}$ & & & & \\
\hline Linalool & $0.635^{* *}$ & $0.654^{* *}$ & $0.7^{* * *}$ & $0.836^{* *}$ & $0.812^{* *}$ & $0.732^{* * *}$ & & & \\
\hline Borneol & $0.619^{* *}$ & $0.691^{* *}$ & $0.764^{* *}$ & $0.634^{* *}$ & $0.635^{* *}$ & $0.546^{* *}$ & $0.831^{* *}$ & & \\
\hline Thymol & $-0.606^{* *}$ & $-0.641^{* *}$ & $-0.591^{* *}$ & $-0.790^{* *}$ & $-0.887^{* *}$ & $-0.816^{* *}$ & $-0.674^{* *}$ & $-0.437^{*}$ & \\
\hline Caryophylene & $-0.761^{* *}$ & $-0.786^{* *}$ & $-0.669^{* *}$ & $-0.893^{* *}$ & $-0.892^{* *}$ & $-0.837^{* *}$ & $-0.859^{* *}$ & $-0.745^{* *}$ & $\mathbf{0 . 7 3 3}^{* *}$ \\
\hline
\end{tabular}

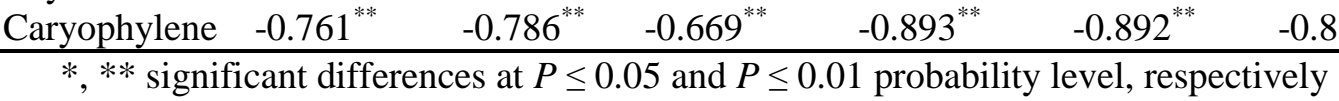

$-0.859^{*}$

$-0.745^{*}$

.733** $^{* *}$ 
Table 6. Comparison interaction of species, superabsorbent and

Thiobacillus on borneol and caryophylene on Thymus vulgaris and Thymus daenensis

\begin{tabular}{lll}
\hline Interactions & Caryophyllene [\%] & Borneol [\%] \\
\hline $\mathrm{V} \times \mathrm{S} 0 \times \mathrm{T} 0$ & $0.9934^{\mathrm{d}}$ & $2.289^{\mathrm{a}}$ \\
$\mathrm{V} \times \mathrm{S} 0 \times \mathrm{T} 0.5$ & $0.9528^{\mathrm{d}}$ & $1.301^{\mathrm{bcd}}$ \\
$\mathrm{V} \times \mathrm{S} 0.5 \times \mathrm{T} 0$ & $0.8876^{\mathrm{d}}$ & $1.281^{\mathrm{bcd}}$ \\
$\mathrm{V} \times \mathrm{S} 0.5 \times \mathrm{T} 0.5$ & $1.124^{\mathrm{d}}$ & $1.621^{\mathrm{abc}}$ \\
$\mathrm{V} \times \mathrm{S} 1 \times \mathrm{T} 0$ & $0.8859^{\mathrm{d}}$ & $1.278^{\mathrm{bcd}}$ \\
$\mathrm{V} \times \mathrm{S} 1 \times \mathrm{T} 0.5$ & $0.9458^{\mathrm{d}}$ & $1.849^{\mathrm{ab}}$ \\
$\mathrm{D} \times \mathrm{S} 0 \times \mathrm{T} 0$ & $2.351^{\mathrm{c}}$ & $1.081^{\mathrm{cde}}$ \\
$\mathrm{D} \times \mathrm{S} 0 \times \mathrm{T} 0.5$ & $3.112^{\mathrm{bc}}$ & $0.9007^{\mathrm{cde}}$ \\
$\mathrm{D} \times \mathrm{S} 0.5 \times \mathrm{T} 0$ & $2.82^{\mathrm{c}}$ & $0.9435^{\mathrm{cde}}$ \\
$\mathrm{D} \times \mathrm{S} 0.5 \times \mathrm{T} 0.5$ & $3.92^{\mathrm{a}}$ & $0.3738^{\mathrm{e}}$ \\
$\mathrm{D} \times \mathrm{S} 1 \times \mathrm{T} 0$ & $3.715^{\mathrm{ab}}$ & $0.6984^{\mathrm{de}}$ \\
$\mathrm{D} \times \mathrm{S} 1 \times \mathrm{T} 0.5$ & $2.93^{\mathrm{c}}$ & $0.9215^{\mathrm{cde}}$ \\
\hline
\end{tabular}

-Treatment symbols: $\mathrm{T}_{0}, 0.0 \mathrm{~g}$ Thiobacillus; $\mathrm{T}_{0.5}, 0.5 \mathrm{~g}$ Thiobacillus; $\mathrm{S}_{0,} 0.0 \mathrm{~g}$ superabsorbent; $\mathrm{S}_{0.5}, 0.5 \mathrm{~g}$ super absorbent; $\mathrm{S}_{1}, 1.0 \mathrm{~g}$ superabsorbent; $\mathrm{V}$, Thymus vulgaris; $\mathrm{D}$, Thymus daenensis.

-Values with different letters in a column are significantly different according to Duncan's Multiple Range Test $(\mathrm{P} \leq 0.05)$

Figure legends

Figure 1. Caryophyllene and borneol change trend across superabsorbent and Thiobacillus

interaction levels over the Thymus species. Values with different letters in each graph are

Figure 2. Mean comparisons of Thymol under the interaction of species $\times$ Thiobacillus. V

and D present Thymus vulgaris and Thymus daenensis, respectively. Values with different 
1. Leung AY, Foster S (1996) Encyclopedia of common natural ingredients used in food, drugs, and cosmetics. John Wiley \& Sons, Inc DOI 10.1021/np960461r

2. Abdulkarimi R, Daneshyar M, Aghazadeh A (2011) Thyme (Thymus vulgaris) extract consumption darkens liver, lowers blood cholesterol, proportional liver and abdominal fat weights in broiler chickens, volume 10, Issuse 2, Ital $\mathrm{J}$ Animal Sci DOI.org/10.40801/ijas.2011.e20

3. Satyal P, Murray BL, Mcfeeters RL, Setzer WN (2016) Essential Oil Characterization of Thymus vulgaris from Various Geographical Locations. Food J 5(4), 70, DOI 10.3390/foods5040070

4. Nickavar B, Mojab F, Dolat-Abadi R (2005) Composition of the Volatile Oil of Thymus daenensis Celak. subsp. daenensis. J Med Plants 1:45-49

5. Intodia SK, Sahu MP (2005) Effect of sulphur fertilization on growth of opium poppy in calcareous soils of south Rajasthan. Indian J Plant Physiol 10:90-93

6. Esmaeeli A, Amiri H (2006) The study of quantitative and qualitative changes of essential oil from Smyrnium cordifolium boiss. In lorestan province. J Med. Plants $5: 36-41$

7. Naghdi badi H, Makizadeh tafti M. (2002) Review of common thyme. J Med. Plants $2: 1-12$

8. Barazandeh M, Bagherzadeh K (2007) Investigation on the Chemical Composition of the Essential of Thymus daenensis Celak from Four Different Regions of Isfahan Province. J Med. Plants 3:15-19

9. Mehnaz S, Lazarovits G (2006) Inoculation effects of Pseudomonas putida, Gluconacetobacter azotocaptans, and Azospirillum lipoferum on corn plant growth under greenhouse conditions. Microb Ecol 51:326-335 DOI 10.1007/s00248-0069039-7

10. Sharafzadeh Sh and Ordookhani, K (2011) Organic and Bio Fertilizers as a Good Substitute for Inorganic Fertilizers in Medicinal Plants Farming. Aust J Basic Appl Sci 5(12); 1330-1333

11. Kumari B, Hora A, Mallick MA (2017) Stimulatory effect of PGPR (Plant Growth Promoting Rhizospheric Bacteria) on Medicinal and Growth properties of a potential medicinal herb Chlorophytum borivilianum: A review. J Plant Sci Res 33(2): 151-156

12. Yadegari M, Barzegar R (2010) The effect of sulphur and Thiobacillus on nutrient absorption, growth and essential oil in lemon balm (Melissa officinalis L.). J Herbal Drugs 1:28-32

13. Besharati H, Saleh rastin N, Malakouti MJ (2004) Study of thiobacilli survival on different carriers. Iranian J Soil Waters Sci 18:170-181

14. Banj Shafiei A, Eshaghi Rad J, Alijanpour A, Pato M (2012) Effects of superabsorbent application and irrigation period on the growth of pistachio seedlings (Pistacia atlantica), (Case study: Dr. Javanshir nursery, Piranshahr). Iranian J Forest 4(2): 101-112

15. Lazcano-Ferrat I, Lovatt CJ (1999) Relationship between Relative Water Content, Nitrogen Pools, and Growth of Phaseolus vulgaris L. and P. acutifolius A. Gray during Water Deficit. Crop Sci DOI 10.2135/cropsci1999.0011183X0039000200028x

16. Razban M, Pirzad AR (2012) Evaluate the effect of varying amounts of super absorbent under different irrigation regimes on growth and water deficit tolerance of german chamomile (Matricaria chamomilla), as a second crop. J Sustain Agricult Prod Sci 21(2): 123-137

17. Zohuriaan-Mehr MJ and Kabiri K (2008) Superabsorbent Polymer Materials: A Review. Iranian Polymer J 17(6): 451-477 
18. Shahhoseini R, Saeidi K, Babaahmadi H, Ebadi MT (2018) Effect of Fertilizers and Superabsorbent Hydrogel on the Yield, Essential Oil Content and Composition of Lemon verbena (Lippia citriodora Kunth.) Cultivated in Iran. J Essential Oil Bearing plants. 21:1, 230-236, DOI: 10.1080/0972060X.2017.1422808

19. Brown DE \& Walton NJ (1999) Chemicals from plants: perspectives on plant secondary products. Imperial College Press, London

20. Al-Asmari AK, Athar MdT, Al-Faraidy AA, Almuhaiza MS (2017) Chemical composition of essential oil of Thymus vulgaris collected from Saudi Arabian market. Asian Pac J Tropic Biomed 7(2): 147-150

21. Charles DJ and Simon JE (1990) Comparison of Extraction Methods for the Rapid Determination of Essential Oil Content and Composition of Basil. J American Soc Horticult Sci 115(3): 458-462

22. Rahimzadeh S, Sohrabi Y, Pirzad A, Sheykhbaglou R (2017) Effect of Biological and Chemical Fertilization on the Yield and Nutrients of Moldavian Balm 

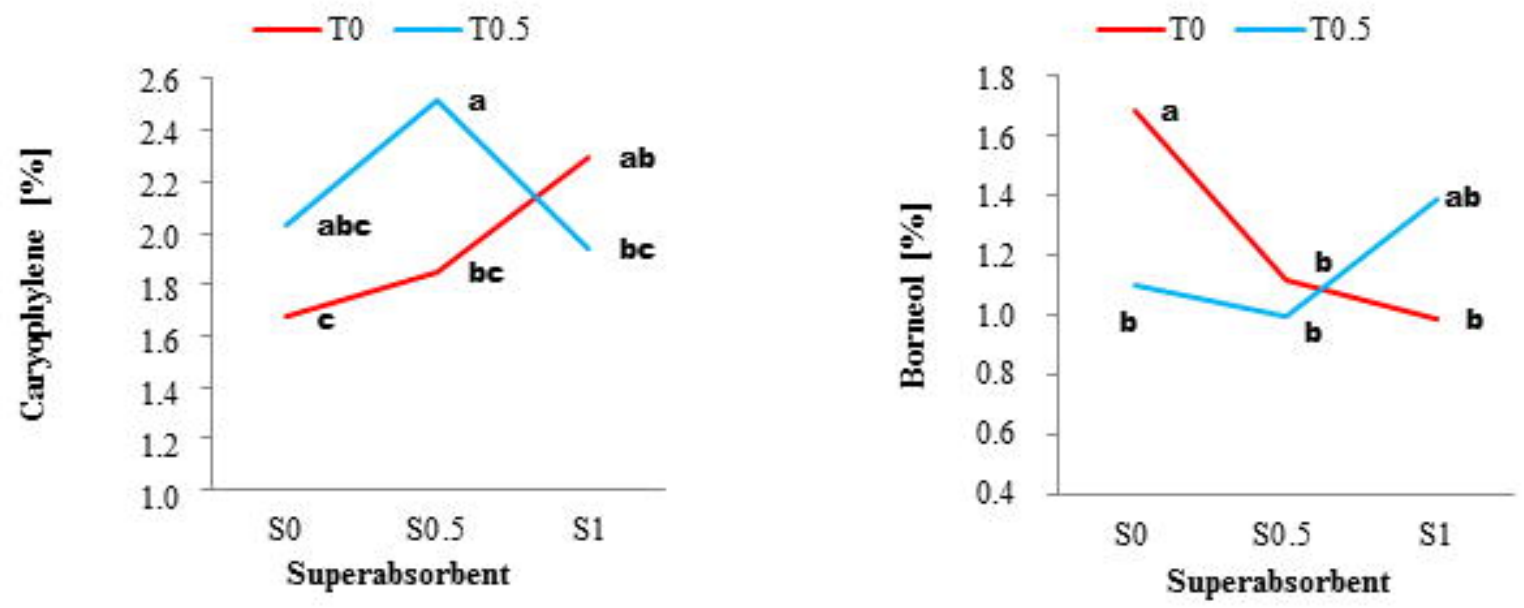

Figure 1. Caryophyllene and borneol change trend across superabsorbent and Thiobacillus interaction levels over the Thymus species. Values with different letters in each graph are significantly different according to Duncan's Multiple Range Test $(\mathrm{P} \leq 0.05)$. 


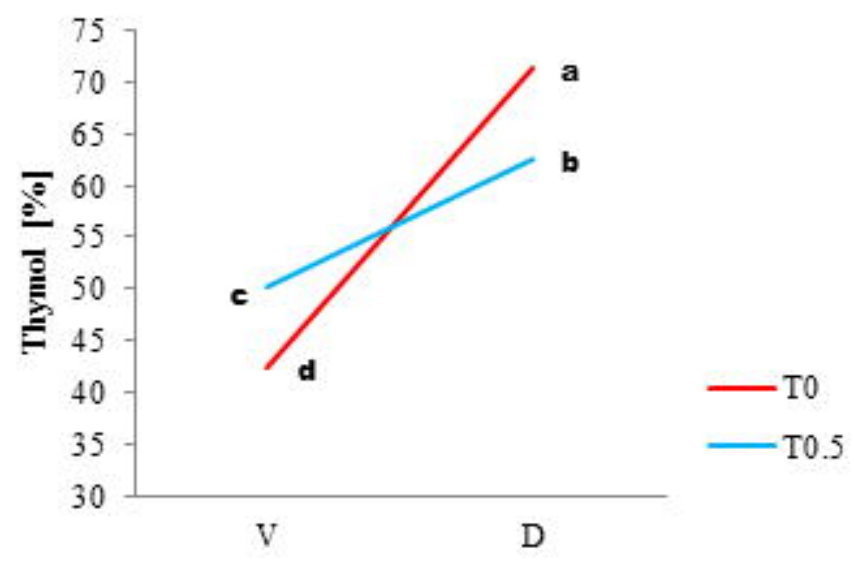

Figure 2. Mean comparisons of thymol under the interaction of species $\times$ Thiobacillus. $\mathrm{V}$ and $\mathrm{D}$ present Thymus vulgaris and Thymus daenensis, respectively. Values with different letters are significantly different according to Duncan's Multiple Range Test $(\mathrm{P} \leq 0.05)$. 